\title{
First principles calculation on the structure and electronic properties of BNNTs functionalized with isoniazid drug molecule
}

\author{
Nabanita Saikia · Swapan K. Pati • Ramesh C. Deka
}

Received: 18 November 2011/ Accepted: 17 April 2012/Published online: 13 May 2012

(c) The Author(s) 2012. This article is published with open access at Springerlink.com

\begin{abstract}
One-dimensional nanostructures such as nanowires and nanotubes are stimulating tremendous research interest due to their structural, electronic and magnetic properties. We perform first principles calculation using density functional theory on the structural, and electronics properties of BNNTs adsorbed with isoniazid (INH) drug via noncovalent functionalization using the GGA/PBE functional and DZP basis set implemented in SIESTA program. The band structure, density of states and projected density of states (PDOS) plots suggest that isoniazid prefers to get adsorbed at the hollow site in case of $(5,5)$ BNNT, whereas in $(10,0)$ BNNT it favours the bridge site. The adsorption energy of INH onto $(5,5)$ BNNT is smaller than in $(10,0)$ BNNT which proposes that $(10,0)$ BNNT with a larger radius compared to $(5,5)$ BNNT is more favourable for INH adsorption as the corresponding distortion energy will also be quite lower. Functionalization of $(5,5)$ and $(10,0)$ BNNTs with isoniazid displays the presence of new impurity states (dispersionless bands) within the HOMO-LUMO energy gap of pristine BNNT leading to an increase in reactivity of the INH/BNNT system and lowering of the energy gap of the BNNTs. The PDOS plots show the major contribution
\end{abstract}

Electronic supplementary material The online version of this article (doi:10.1007/s13204-012-0124-6) contains supplementary material, which is available to authorized users.

\section{N. Saikia · R. C. Deka $(\square)$}

Department of Chemical Sciences, Tezpur University,

Napaam, Tezpur 784028, Assam, India

e-mail: ramesh@tezu.ernet.in

\section{S. K. Pati}

Theoretical Sciences Unit and New Chemistry Unit, Jawaharlal Nehru Centre for Advanced Scientific Research, Jakkur Campus, Bangalore 560064, India

e-mail: pati@jncasr.ac.in towards the dispersionless impurity states is from INH molecule itself rather than from BNNT near the Fermi energy region. To summarize, noncovalent functionalization of BNNTs with isoniazid drug modulates the electronic properties of the pristine BNNT by lowering its energy gap with respect to the Fermi level, as well as demonstrating the preferential site selectivity for adsorption of isoniazid onto the nanotube sidewalls of varying chirality.

Keywords BNNTs · Isoniazid · DFT · Electronic structure $\cdot$ Wavefunction

\section{Introduction}

In complete analogy to carbon nanotubes (CNT) which are quasi-one dimensional nanomaterials exhibiting unique structural based electronic properties depending on the nanotube diameter and chirality, boron nitride nanotubes (BNNTs) are wide band gap semiconductors (band gap of $\sim 3.5-5.5 \mathrm{eV}$ ) the electronic property of which is independent of the nanotube diameter and helicity and wall number (Rubio et al. 1994; Blase et al. 1994). The BNNTs have been predicted by theoretical calculations and first synthesized experimentally (Chopra et al. 1995) using plasma-induced arc discharge technique. BNNT possesses high mechanical, thermal and chemical stability in addition to the strong resistance towards oxidation (Golberg et al. 2001). BNNTs of high purity and quality are inherently non cytotoxic whereas CNT are quite cytotoxic suggesting BNNT to be suitable for biomedical applications compared to CNT (Chen et al. 2009). The biocompatibility of BNNT is achieved by means of chemical functionalization (Zhi et al. 2008), with enhanced solubility for its practical application in nanoelectronics, biological and biomedical sciences. 
Just as in carbon nanotubes, both covalent (Zhi et al. 2006) and noncovalent functionalization schemes have been adopted for solubilizing BNNTs (Gou et al. 2010; Wang et al. 2008). In case of noncovalent functionalization of BNNT with aromatic molecules, the aromatic ring of the molecule mitigates the $\pi-\pi$ stacking between the two interacting units (Zhao and Ding 2010). Compared to covalent functionalization, noncovalent functionalization is of major theoretical interest because the electronic property and the delocalized network of the nanotube are retained. The noncovalent functionalization of BNNTs with flavin mononucleotides showed strong $\pi-\pi$ stacking between flavin molecule and BNNT sidewall, and fluorescence spectra demonstrated the nanohybrids to be highly fluorescent in the visible spectra (Gao et al. 2011). Density functional theory (DFT) study on the noncovalent functionalization of BNNT with benzaldehyde and seven different heterocyclic aromatic molecules showed that weak interaction between the two interacting units can give rise to new impurity states within the band gap of pristine BNNTs suggesting the possibility of carrier doping via selective adsorption of aromatic rings (Akdim et al. 2009). The DFT study on noncovalent adsorption of perylene derivatives on BNNT was carried out along with the subsequent charge transfer from the adsorbed molecule and BNNT (Gou et al. 2010). The physisorption of nucleobases on BNNT was studied using DFT (LDA) methodology (Mukhopadhyay et al. 2010) which explored the potential biomedical applications of BNNT as the promising candidate especially for biosensor devices. Polymer wrapping facilitates the BNNT dispersion in aqueous solvents and, in turn, the biocompatibility and its interaction with the human cells upon internalization. The interaction of DNA/ RNA nucleobases with carbon and BNNTs is basically governed by the van der Waals interaction and the strength of the interaction depends on the polarizability of nucleobases.

With an aim of expanding the domain of BNNT for drug delivery applications, we have carried out systematic study on the noncovalent functionalization of BNNT with isoniazid antitubercular drug molecule. The interest in theoretical study of INH antitubercular drugs stems from the fact that tuberculosis is one of the most deadly infectious diseases, with a death toll of over 2 million lives per year (Dessen et al. 1995). About 70-90 \% people suffering from AIDS die due to tuberculosis and new drug resistant strains of Mycobacterium tuberculosis usually resists treatment of the disease. Isoniazid forms one of the most potent antitubercular drugs effective against $M$. tuberculosis which functions in combination with pyrazinamide, rifampicin and ethambutol drugs (Scroeder et al. 2002; Favila et al. 2007). Since SWNT as well as MWNTs have been widely explored both experimentally and theoretically in cancer chemotherapy through this work, we have proposed a new strategy of using BNNTs as carrier module in tuberculosis chemotherapy by which target-specific delivery of antitubercular drugs at specific receptor sites within the body can be envisaged retaining the toxicity and physiological characteristics of the therapeutics. In case of CNT, preliminary studies show pristine nanotubes to be toxic while functionalized SWNTs exhibit good biocompatibility within the body. The adsorption, distribution, metabolism, excretion, and toxicity of BNNT are highly dependent on the extent of functionalization, coating and length of the tube (Helland et al. 2007). Through this work, we intend to address the following issues: (1) the stable geometry and adsorption energies for noncovalent interaction of isoniazid onto $(5,5)$ and $(10,0)$ BNNT sidewalls; (2) modulation in electronic properties of pristine nanotube on adsorption of drug molecule in terms of variation in band structure, density of states (DOS), Fermi energy; (3) wavefunction plots corresponding to the electronic charge distribution in drug-nanotube system in terms of HOMO and LUMO orbitals, (4) charge transfer between isoniazid drug molecule and BNNT via non-covalent functionalization at both the parallel and perpendicular orientations of adsorption.

\section{Computational details}

The ab initio spin polarized total energy DFT calculations have been adopted in this study. The generalized gradient approximation (GGA) within the Perdew Burke Ernzerhof (PBE) is used to describe the exchange correlation energy (Pewdew et al. 1996). As it is well known that local density approximation (LDA) produces electronic densities more homogeneous than the exact ones which leads to the overestimation of the binding energy values more predominant in case of weakly bound systems it is believed that for molecular systems, GGA significantly improves the binding (adsorption) energy values. Although conventional DFT methods like LDA and GGA cannot account for dispersive interactions like $\pi-\pi$ stacking and van der Waals interaction, yet we can get a qualitative generalized overview towards the noncovalent functionalization of organic therapeutic molecules with BNNTs of varying chirality indices and diameter. Troullier Martins type pseudopotentials (Troullier and Martins 1991) in the KleinmanBylander form (Kleinman and Bylander 1982) and DZP basis set are used to describe the valence state wavefunction in the reciprocal space within a plane-wave cutoff of $300 \mathrm{Ry}$. The radial part of the basis function is described using the zeta function. To sample the Brillouin Zone (BZ), a set of 60 Monkhorst-Pack (Monkhorst and Pack 1976) special k-points are used along the nanotube axis with a vacuum separation of about $20 \AA$ to avoid the lateral 
interactions between the replicating periodic supercell images. The conjugate gradient (CG) method is employed to perform the geometry optimization and the system is relaxed until the residual forces of all the atoms are converged below $0.03 \mathrm{eV} / \AA$.

To investigate the interaction of isoniazid drug molecule with BNNTs two nanotubes of varying chirality namely, armchair $(5,5)$ and zigzag $(10,0)$ BNNTs each comprising of 100 and 120 atoms, respectively, are adopted in the simulation. The isoniazid adsorption onto the two nanotubes was studied for two modes of adsorption, namely the parallel and perpendicular orientations. For the parallel orientation of adsorption, the interaction was studied for four basic adsorption sites: (a) hollow, (b) bridge, (c) B top and (d) $\mathrm{N}$ top sites; the pictorial description of which is given in Fig. 1. The band structure, DOS, projected density of states (PDOS) and wavefunction of the isosurface corresponding to HOMO and LUMO are also calculated using the SIESTA method. The adsorption energy is defined as $E_{\text {ads }}=E_{\text {(complex) }}-E_{(\mathrm{BNNT})}-E_{(\mathrm{INH})}$, where $E_{\text {(complex) }}$ is the total energy of the system (BNNT + INH). A more negative $E_{\text {ads }}$ value denotes more favourable interaction between the drug-nanotube systems.

\section{Results and discussion}

Structural properties and adsorption energies

Figure 1 depicts the four basic adsorption sites: (A) hollow, (B) bridge, (C) $\mathrm{N}$ top and (D) B top sites for the stacking interaction of INH onto $(5,5)$ and $(10,0)$ BNNTs, respectively. As observed from the figure, in the hollow site (A) the pyrazine ring is placed directly above the $\mathrm{BN}$ ring of the BNNT, whereas in the bridge site of adsorption (B) the pyrazine ring is placed between the $\mathrm{BN}$ bonds represented by the black square of the Fig. 1. The B top and $\mathrm{N}$ top sites denote the positions where the pyrazine ring is placed above these two sites i.e. immediately above the black squares.

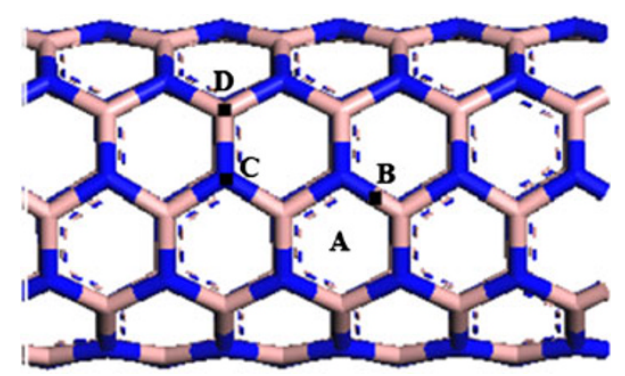

Fig. 1 The four basic adsorption sites of INH onto $(5,5)$ and $(10,0)$ BNNTs: $A$ hollow, $B$ bridge, $C \mathrm{~N}$ top and $D$ B top sites
The optimized geometry of isoniazid (Fig. 2a) suggests it to be a planar molecule with the amine group projecting out of the molecular plane. It is a $\pi$ electronic system with the $\pi$ states contributed from the HOMO and the $\pi^{*}$ states the corresponding LUMO.

The band gap of isoniazid is calculated as $3.331 \mathrm{eV}$. The $\pi$ and $\pi^{*}$ states in isoniazid are basically composed from the $p_{\mathrm{z}}$ atomic orbitals of $\mathrm{C}$ atom of the pyrazine ring and the carboxyl group can provide the extra delocalized $\pi$ electrons leading to an enhanced conjugation within the drug molecule. The optimized geometries of pristine $(5,5)$ and $(10,0)$ BNNT are shown in Fig. 2b, c, respectively. Five unit cells of $(5,5)$ BNNT and four unit cells of $(10,0)$ BNNT are taken for the study to investigate its interaction with INH at both parallel and perpendicular adsorption sites. The B-N bond length is calculated to be $1.45 \AA$ after complete structure relaxation.

The four initial positions for adsorption of INH onto the outer sidewall of $(5,5)$ and $(10,0)$ BNNTs are considered namely $\mathrm{B}$ top, $\mathrm{N}$ top, hollow and bridge sites, followed by the perpendicular adsorption to determine the most favourable orientation. The geometries corresponding to the most preferred site of parallel adsorption of INH onto $(5,5)$ are shown in Fig. 3a (front view) and b (side view) and for $\mathrm{INH} /(10,0)$ BNNT the adsorption orientation is shown in Fig. 3d, e, corresponding to the front and side views. The perpendicular adsorption of INH onto $(5,5)$ and $(10,0)$ BNNTs is shown in Fig. 3c, f, respectively.

To evaluate the preferred interaction, we calculated the adsorption energy of INH onto the two individual nanotubes. The values for the adsorption energies are provided in Table 1 for $\mathrm{INH} /(5,5)$ BNNT, and for $\mathrm{INH} /(10,0)$ BNNTs it is given in Table 2 along with their corresponding distance of interaction.

In case of parallel adsorption of INH onto $(5,5)$ BNNT, the preferred site of adsorption is the hollow site (Fig. 3a) having an adsorption energy $\left(E_{\mathrm{ads}}=-0.649 \mathrm{eV}\right)$ and in $(10,0)$ BNNT it is the bridge site (Fig. 3d) with the adsorption energy $\left(E_{\text {ads }}=-0.738 \mathrm{eV}\right)$ which may be due to the nature of the two different nanotubes. Since neither LDA nor GGA functional can accurately predict the van der Waals interaction (dispersive interaction), the results corresponding to the adsorption energy and HOMOLUMO gap are rather qualitative but can account for the trend in the interaction of the drug molecule on the nanotube sidewalls. The high HOMO-LUMO gap of INH molecule suggests it to be quite stable. After adsorption of INH onto $(5,5)$ BNNT the HOMO-LUMO gap significantly reduces, implying an increase in reactivity of the $\mathrm{INH} /(5,5)$ BNNT system. However, for the perpendicular adsorption of INH, the lowering of the energy gap is by $0.08 \mathrm{eV}$. This also justifies the fact that the parallel adsorption of INH onto $(5,5)$ BNNT is more favoured. The

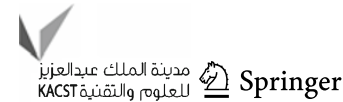


Fig. 2 Optimized structures of a isoniazid, b five unit cells of $(5,5)$ and $\mathbf{c}$ four unit cells of $(10,0)$ BNNT (a)

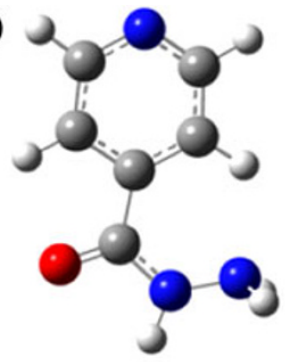

(b)

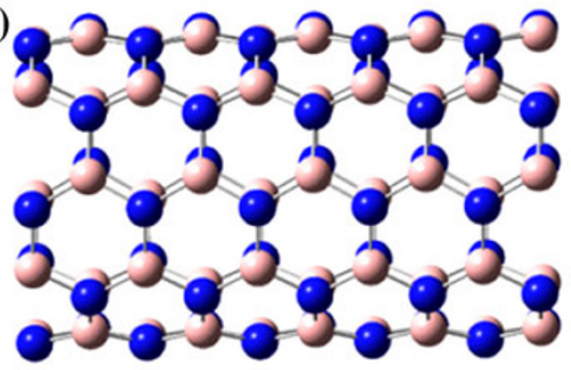

(c)

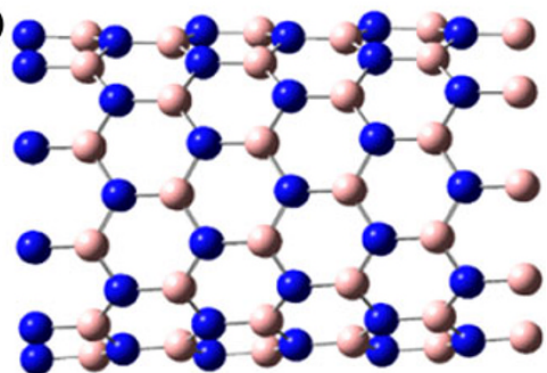

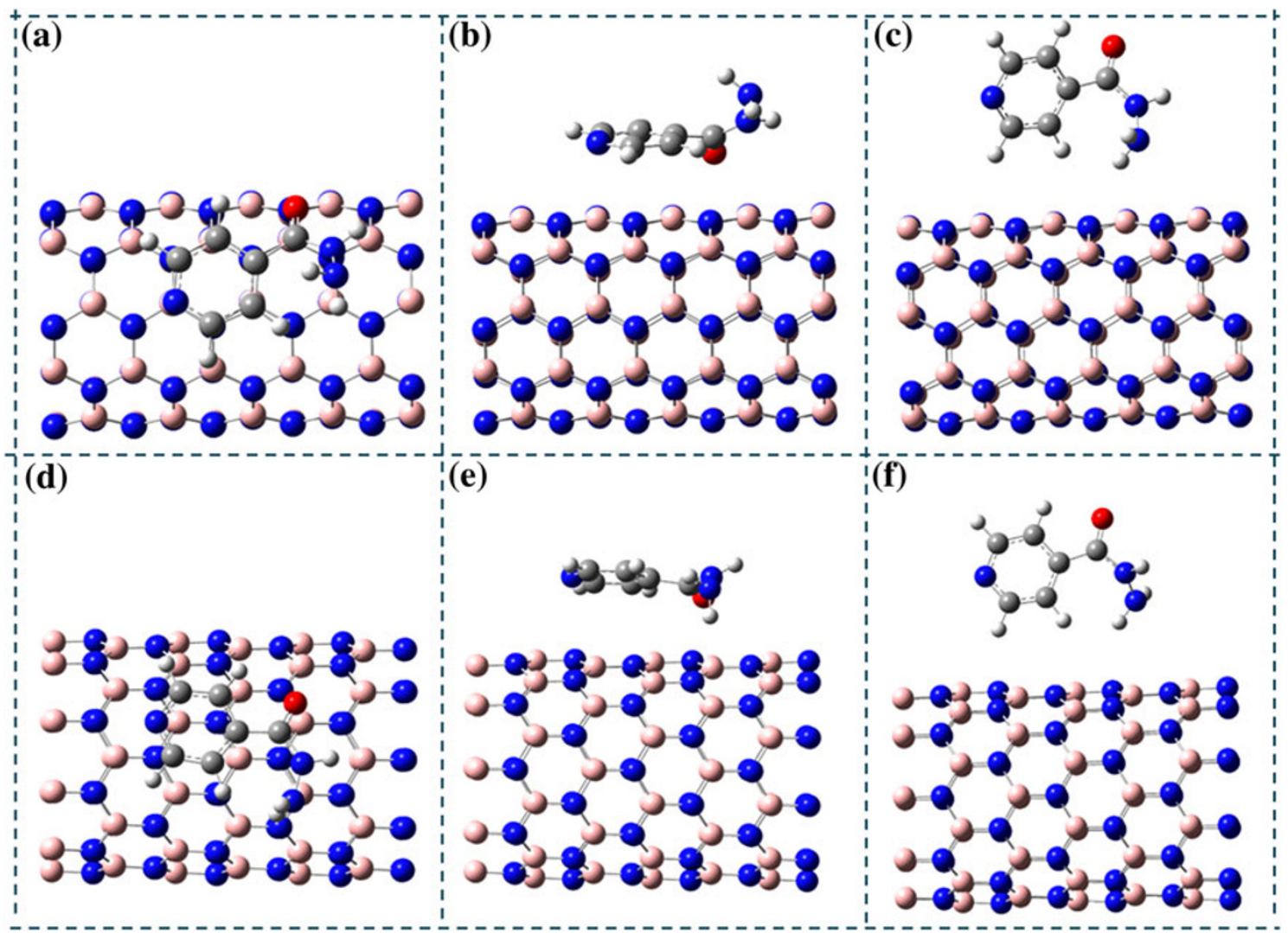

Fig. 3 Optimized structures corresponding to the stable geometries of INH adsorbed onto $(5,5)$ BNNT at the hollow site: a front view, b side view $\mathbf{c}$ perpendicular orientation of adsorption, $\mathbf{d}$ front view of

adsorption energy of INH onto $(5,5)$ BNNT is smaller than in $(10,0)$ BNNT whereas the behaviour of adsorption is similar which suggests that $(10,0)$ BNNT with a larger radius compared to $(5,5) \mathrm{BNNT}$ is more favourable for INH adsorption as the corresponding distortion energy will
INH adsorbed onto $(10,0)$ BNNT at the bridge site, e side view for the same and $\mathbf{f}$ the perpendicular adsorption

also be quite lower in $(10,0)$ BNNT. Thus, the radius of the nanotube can play a significant role in the adsorption and interaction of small organic aromatic molecules with nanotube sidewalls. Since BNNT is ionic in character, more electrons get distributed over the $\mathrm{N}$ atoms compared 
Table 1 Adsorption energies $(\mathrm{eV})$, Fermi energy (eV), equilibrium distance of interaction $(\AA)$ and HOMOLUMO energy gap $(\mathrm{eV})$ of INH/(5,5) BNNT system

Table 2 Adsorption energies $(\mathrm{eV})$, Fermi energy $(\mathrm{eV})$, equilibrium distance of interaction $(\AA)$ and HOMOLUMO energy gap $(\mathrm{eV})$ of $\mathrm{INH} /(10,0)$ BNNT system

\begin{tabular}{lllll}
\hline System & $\begin{array}{l}\text { Adsorption } \\
\text { energy }(\mathrm{eV})\end{array}$ & $\begin{array}{l}\text { Distance of } \\
\text { interaction }(\AA)\end{array}$ & $\begin{array}{l}\text { Fermi } \\
\text { energy }(\mathrm{eV})\end{array}$ & $\begin{array}{l}\text { HOMO-LUMO } \\
\text { gap (eV) }\end{array}$ \\
\hline (5,5) BNNT & & & -3.344 & 4.928 \\
INH & & & -3.662 & 3.331 \\
INH/(5,5) BNNT bridge & -0.539 & 3.169 & -4.774 & 3.173 \\
INH/(5,5) BNNT hollow & -0.649 & 3.138 & -4.857 & 3.174 \\
INH/(5,5) BNNT N top & -0.601 & 3.166 & -4.801 & 3.205 \\
INH/(5,5) BNNT B top & -0.566 & 3.226 & -4.771 & 3.163 \\
INH/(5,5) BNNT perp site & -0.098 & 2.957 & -4.420 & 3.251 \\
\hline & & & & \\
\hline System & Adsorption & Distance of & Fermi & HOMO-LUMO \\
& energy (eV) & interaction $(\AA)$ & energy (eV) & gap (eV) \\
\hline (10,0) BNNT & & & -5.129 & 3.932 \\
INH & & & -3.662 & 3.331 \\
INH/(10,0) BNNT bridge & -0.738 & 3.311 & -4.848 & 3.049 \\
INH/(10,0) BNNT hollow & -0.702 & 3.185 & -4.769 & 3.026 \\
INH/(10,0) BNNT N top & -0.691 & 3.158 & -4.803 & 3.139 \\
INH/(10,0) BNNT B top & -0.655 & 3.243 & -4.760 & 3.069 \\
INH/(10,0) BNNT perp site & -0.391 & 2.663 & -4.200 & 2.442 \\
\hline
\end{tabular}

to $\mathrm{B}$ atoms. Hence on adsorption of aromatic ring onto BNNT sidewall, the ring-like delocalized electron near the molecular plane tends to minimize the electronic repulsion between the BNNT, so that the centre of the ring prefers to be over the hollow site which is the case in $\operatorname{INH} /(5,5)$ BNNT system.

The negative values of adsorption energies corresponding to INH adsorption onto $(5,5)$ and $(10,0)$ BNNT suggest that the adsorption to be exothermic and experimentally feasible from the energetic perspective and INH molecule is stabilized by the BNNT surface. Adsorption of INH onto $(10,0)$ BNNT displays a similar lowering of HOMOLUMO energy gap as observed in case of $(5,5)$ BNNT. The lowering of the energy gap in both the studied $(5,5)$ and $(10,0)$ BNNTs shows that functionalization increases the reactivity (lowers the stability) of the pristine nanotubes. Although GGA underestimates the binding energy values and HOMO-LUMO energy gap for noncovalent functionalization, the basic mechanism and the physical essence behind the interaction do not change, because of which GGA methodology can be used for validating our study.

Electronic band structure, DOS and PDOS analysis

The electronic band structure of isoniazid, (5,5) BNNT, $(10,0)$ BNNT is shown in Fig. 4, and the corresponding INH/(5,5) BNNT and INH/(10,0) BNNT systems displayed in Figs. 6 and 7, respectively. In $(5,5)$ BNNT, the Fermi level of the semiconducting nanotube is calculated to be $-3.344 \mathrm{eV}$ (Fig. 4b) which upon adsorption of isoniazid gets lowered as given in Table 1. The HOMO-LUMO gap in $(5,5) \mathrm{BNNT}$ is calculated as $4.93 \mathrm{eV}$ at the $\Gamma$ point. In pristine $(5,5) \mathrm{BNNT}$, the HOMO and LUMO states are equally dispersed with respect to the Fermi level (Fig. 4b), whereas in pristine (10,0) BNNT (Fig. 4c) the HOMO states are more close to the Fermi level compared to the LUMO such that it can easily contribute electronic states above the Fermi level. The LUMO on the other hand is located far away from the Fermi region. Although spin polarized calculations were initially performed, the up and down spin electronic states are the same as observed from the figure, the pristine BNNTs, INH molecule are found to be spin unpolarized.

The DOS and PDOS plots of pristine $(5,5)$ and $(10,0)$ BNNTs are displayed in Fig. 5. The DOS plots and the band gap are in agreement with the band structure plots of pristine $(5,5)$ and $(10,0)$ BNNTs. The PDOS plots for $(10,0)$ BNNT (Fig. 5b) show that the B2p orbitals have the major contribution towards the PDOS in the conduction band region above the Fermi level, whereas in the valence band region $\mathrm{N} 2 \mathrm{p}$ orbitals constitute the major states. The $\mathrm{N} 2 \mathrm{~s}$ and B2s orbitals are very low lying electronic states and do not have any major contribution. The same holds in case of $(5,5)$ BNNT as observed in Fig. 5c, d. The PDOS plots for both the studied BNNTs suggest that the electronic states in the valence band region are basically from the $\mathrm{N} 2 \mathrm{p}$ orbitals and in the conduction band region it is from the B2p orbitals.

Upon adsorption of isoniazid onto $(5,5)$ BNNT at the hollow site, the band structure plots show the presence of new dispersionless bands comprising mainly from the interaction of INH with the nanotube around 2.52 and 

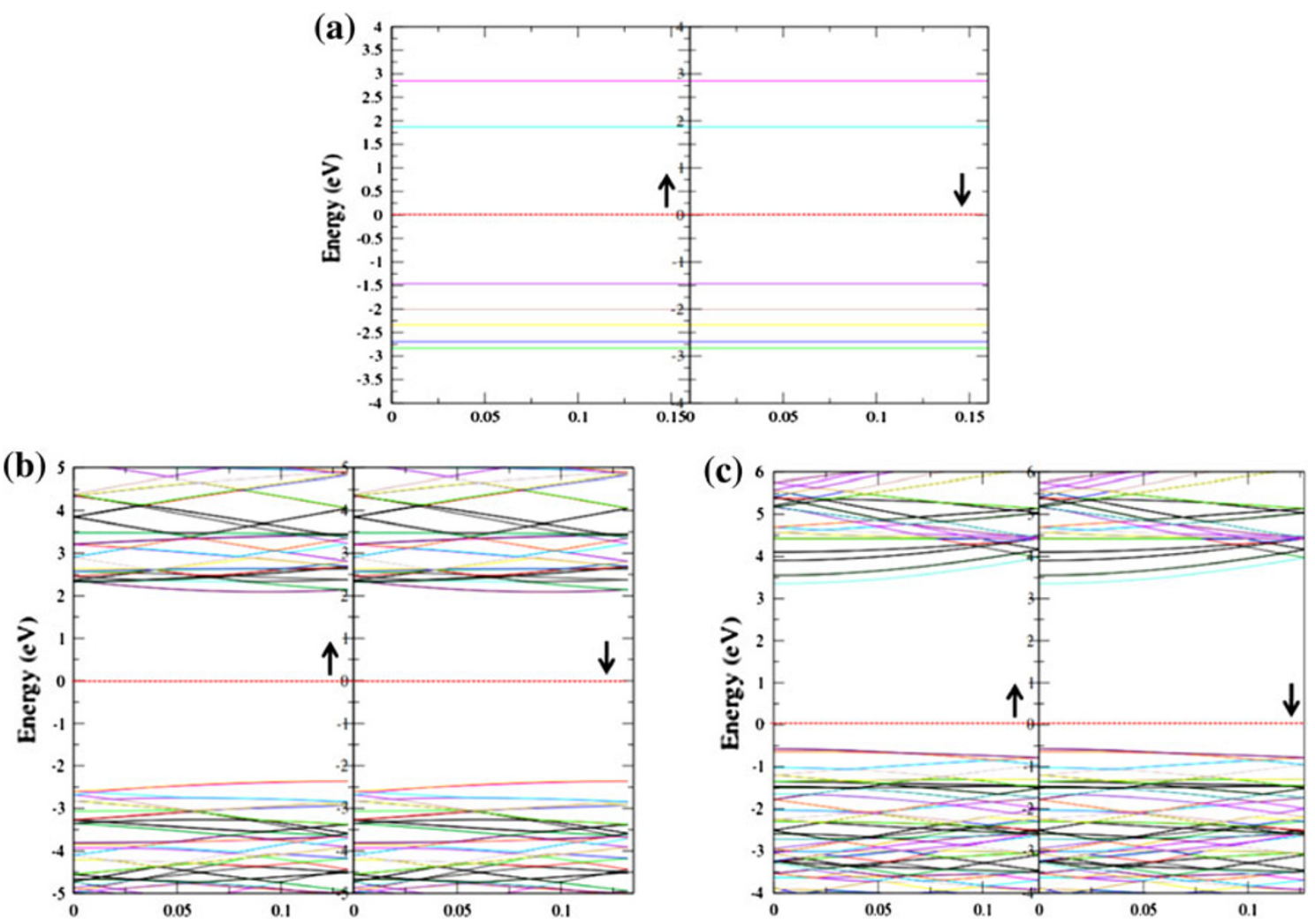

Fig. 4 The band structures of isoniazid molecule (a), pristine $(5,5)$ BNNT (b) and pristine $(10,0)$ BNNT (c). The Fermi energy level is set to zero and indicated by the red dash line. The up and down arrows denote the up and down spins, respectively

$3.36 \mathrm{eV}$ in the conduction band and $-0.61 \mathrm{eV}$ in the valence band region of $(5,5)$ BNNT system (Fig. 6 top). It is quite interesting to note here that in $\mathrm{INH} /(5,5) \mathrm{BNNT}$ system the electronic states coming from the valence band region moves up with respect to the Fermi level and the conduction band electronic states move more deeper in the energy band structure. Similar trend is observed for all the studied modes of adsorption of INH onto $(5,5)$ BNNT including the perpendicular orientation of adsorption. For clarity, we have only shown the band structure, DOS, and PDOS plots of INH/(5,5) SWNT system with INH adsorbed at the hollow site (Fig. 6 top) and INH adsorbed at the perpendicular site (Fig. 6 bottom), respectively.

The band structure, DOS and PDOS plots of INH adsorbed onto $(5,5) \mathrm{BNNT}$ at the bridge, $\mathrm{N}$ top and $\mathrm{B}$ top sites are given in the Supporting Information (SI, Figs. S1S3). It is observed that for the parallel orientation of adsorption of INH onto $(5,5)$ BNNT the band structure, DOS, and PDOS plots are almost same and the newly formed dispersionless bands are situated in the same energy regions. The valence band region corresponding to the HOMO moves upward with respect to the Fermi level and the conduction band region also shows a relative shift. For the perpendicular adsorption of INH onto $(5,5)$ BNNT (Fig. 6 bottom), the effect of INH adsorption on the electronic structure of BNNT is quite different with a single dispersionless band at $\sim 2.53 \mathrm{eV}$ in the conduction band region and three dispersion less bands in the region of -0.5 to $-1.25 \mathrm{eV}$ of the valence band region are observed. Therefore, the band gap decreases in comparison to the pristine $(5,5)$ BNNT by $3.25 \mathrm{eV}$. Thus, in case of INH adsorbed onto $(5,5)$ BNNT (parallel configuration), three impurity states are found within the HOMO-LUMO gap two in the conduction band and one in the valence band region (Fig. 6 top). However, for the perpendicular adsorption of INH onto (5,5) BNNT (Fig. 6 bottom), the pattern is just the reverse with one impurity state in the $\mathrm{CB}$ region and two new impurity states in the $\mathrm{VB}$ region close to the Fermi level.

In pristine $(10,0) \mathrm{BNNT}$, the band gap is calculated to be $3.932 \mathrm{eV}$ at the $\Gamma$ point (Fig. 4c). The parallel adsorption of isoniazid onto $(10,0)$ BNNT demonstrates the presence of dispersionless bands at $2.39 \mathrm{eV}$ in the conduction band region and -0.65 and $-0.848 \mathrm{eV}$ in the valence band region which is accountable from the isoniazid molecule (Fig. 7a). For all the studied orientations of adsorption of INH onto $(10,0)$ BNNT, the bridge site is preferred for adsorption. In pristine $(10,0)$ BNNT, the Fermi energy level is situated close to the valence band region. Upon adsorption of INH, the HOMO-LUMO gap reduces to $\sim 3.02 \mathrm{eV}$ (Table 2). For INH adsorbed onto $(10,0)$ BNNT at the parallel orientation of adsorption (Fig. 7 top), two 
$(10,0)$ BNNT
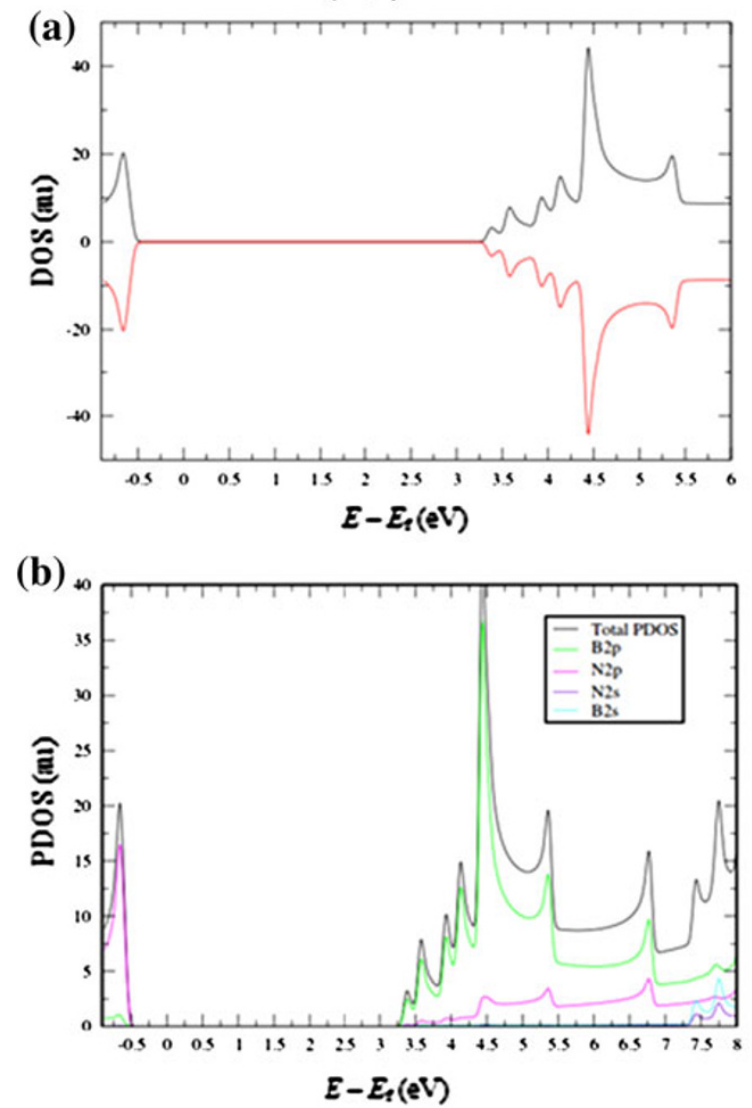

$(5,5)$ BNNT

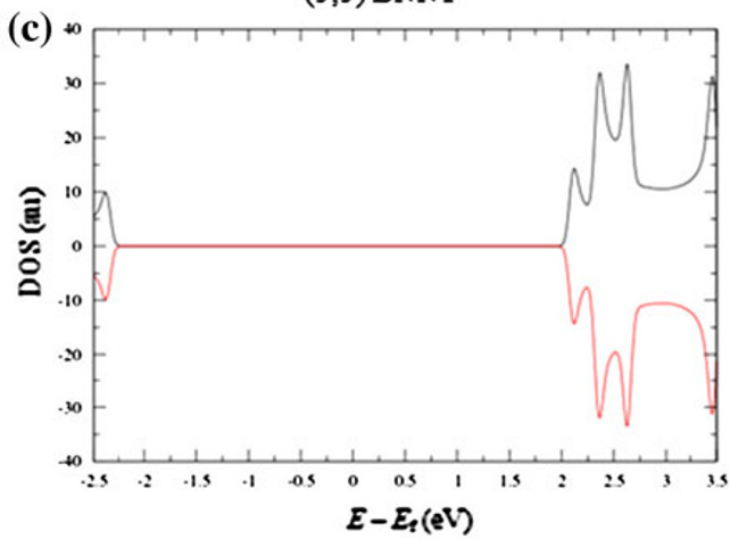

(d)

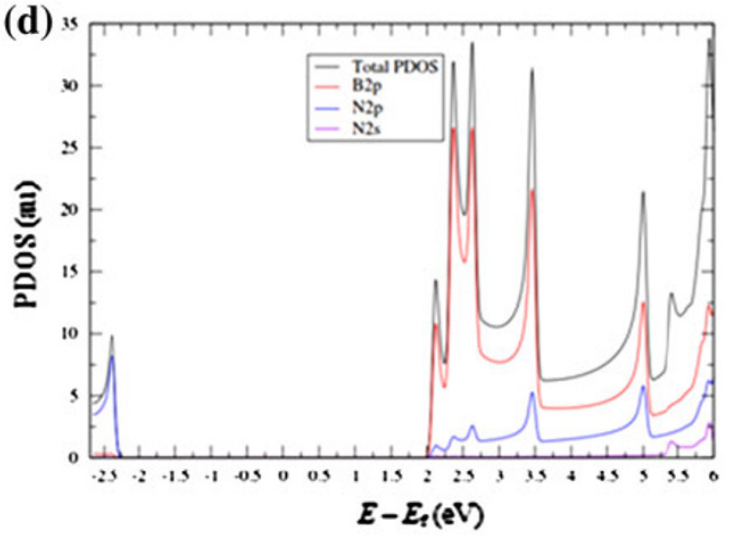

Fig. 5 a The DOS plot of $(10,0)$ BNNT, b the PDOS plot of $(10,0)$ BNNT, c DOS plot of $(5,5)$ BNNT and d PDOS plot of (5,5) BNNT. The Fermi level is set to zero. For the PDOS plots, only the up spin contributions have been shown

impurity states are present within the HOMO-LUMO energy gap one in close proximity to the HOMO of BNNT and the other lying around $2.5 \mathrm{eV}$ in the $\mathrm{CB}$ region and another dispersionless band at $3.35 \mathrm{eV}$, respectively. In the adsorption of INH onto $(10,0)$ BNNT at the perpendicular site (Fig. 7 bottom), three new dispersionless bands are found within the band gap of $(10,0)$ BNNT around -0.5 to $-1.5 \mathrm{eV}$ in the valence band region. Likewise, in the conduction band region around $1.80 \mathrm{eV}$, a new dispersionless band is formed which is also due to the adsorbed INH molecule. For the perpendicular orientation of adsorption, the conduction band (LUMO) moves down with respect to the Fermi level whereas the HOMO level also shifts relative to the Fermi energy. Thus, the band and DOS plots of INH/BNNT systems suggest that there is a significant interaction between the HOMO and LUMO orbitals of INH and the valence and conduction bands of pristine $(5,5)$ and $(10,0)$ BNNT although the extent of interaction is quite different for the two nanotube studied. It is, therefore, expected that charge transfer should preferably occur between the INH molecule and nanotube sidewall. The adsorption of INH onto both $(5,5)$ and $(10,0)$ BNNT is predicted to induce new impurity states within the band gap of pristine BNNT resulting in the reduced band gap of the INH/BNNT system. The Mulliken charge population analysis was also carried out to validate the above findings and it is shown that in $(5,5) \mathrm{BNNT/INH}$ system the charge transfer is almost negligible. The DOS plots confirm the presence of new dispersionless impurity states in the valence and conduction band regions within the HOMOLUMO gap of $(5,5)$ and $(10,0)$ BNNT. On the other hand, the projected DOS (PDOS) plots can provide a detailed understanding to the individual species contribution in the Fermi energy region. The new introduced states in INH/ $(5,5) \mathrm{BNNT}$ and INH/(10,0) BNNT are mainly ascribed to the adsorbed INH drug molecule in the vicinity of the Fermi energy and BNNT has contributions in the valence and $\mathrm{CB}$ regions away from the Fermi level. Hence INH serves as the basic active unit which relates to the electron mobility upon interaction with BNNT.

\section{Wavefunction analysis}

To understand the electronic properties of INH/BNNT system the energy bands around the Fermi level are examined with the help of the wavefunction plots 
$\mathrm{INH} /(5,5)$ BNNT hollow site
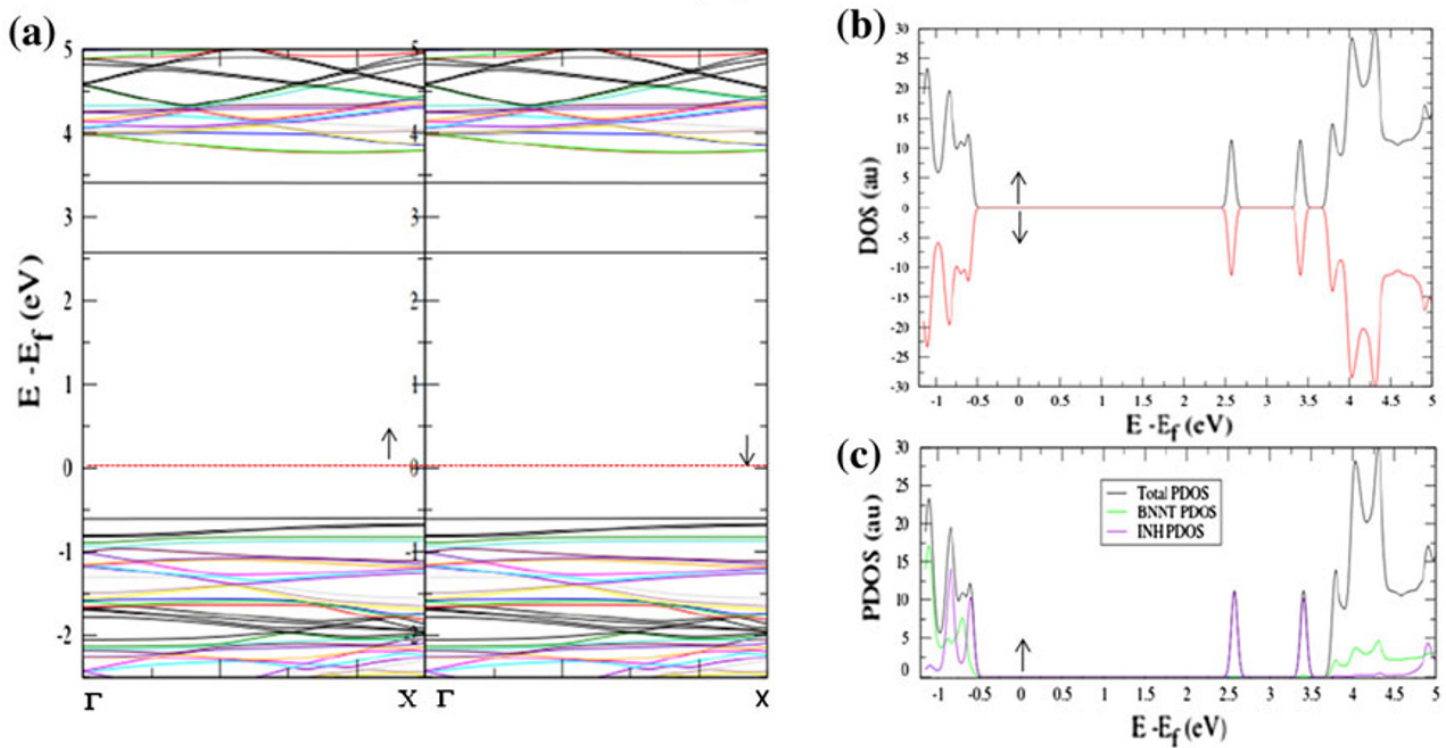

$\mathrm{INH} /(5,5)$ BNNT perpendicular site
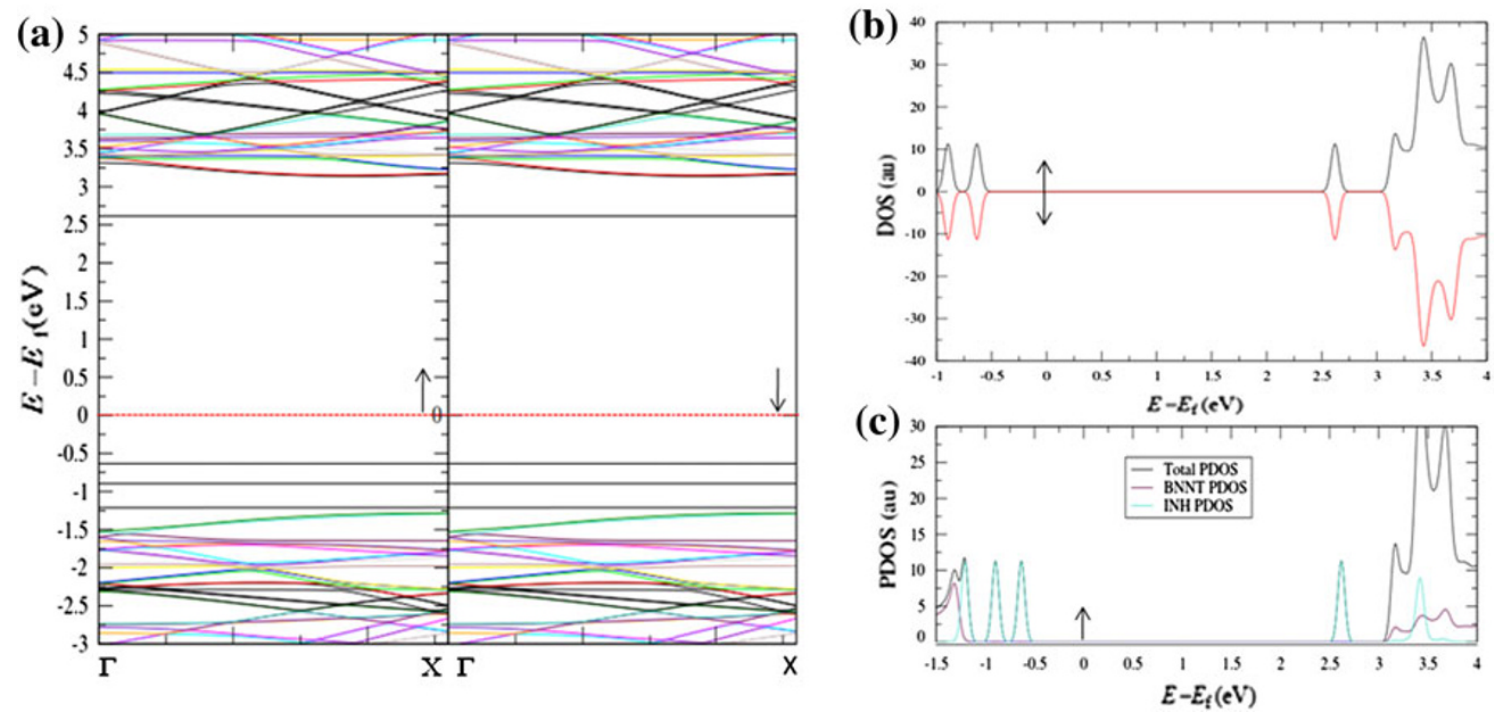

Fig. 6 Top the band structure (a), DOS (b), PDOS (c) plots of INH adsorbed onto $(5,5)$ BNNT at the hollow site, and bottom the band structure (a), DOS (b) and PDOS (c) plots of INH adsorbed onto $(5,5)$

corresponding to the HOMO and LUMO at the $\Gamma$ points. The wavefunction plots for $\operatorname{INH} /(5,5)$ BNNT system is shown in Fig. 8 and INH/(10,0) BNNT system is displayed in Fig. 9. In INH/(5,5) BNNT system with INH adsorbed at the hollow site (Fig. 8a), the HOMO has major contribution from the INH molecule with partial contribution from the nanotube sidewall at the closest proximity of the drug molecule. On the other hand, the LUMO plot (Fig. 8b) is distributed or rather delocalized between the INH molecule and BNNT sidewall, exhibiting electronic charge density to be delocalized between the interacting groups. For the perpendicular adsorption of INH onto $(5,5)$ BNNT, the
BNNT at the perpendicular site. The Fermi energy level is set to zero and indicated by the red dash line. The up and down arrows denote the up and down spins, respectively

HOMO states (Fig. 8c) are basically localized onto the drug molecule, whereas the LUMO is partially distributed onto the nanotube sidewall (Fig. 8d) in close vicinity to the INH molecule. However, in INH adsorbed onto $(5,5)$ BNNT at the B top site, the LUMO is delocalized onto the INH and BNNT, however, it does not imply any hybridization between the orbitals of the interacting groups as opposed to chemisorption. The wavefunction plots for $\mathrm{INH} /(5,5) \mathrm{BNNT}$ at bridge, $\mathrm{N}$ top and B top sites are shown in the Supporting Information (SI, Fig. 2). The wavefunction plots corresponding to the HOMO and LUMO show that INH contributed significant components in the 
INH/(10,0) BNNTbridgesite
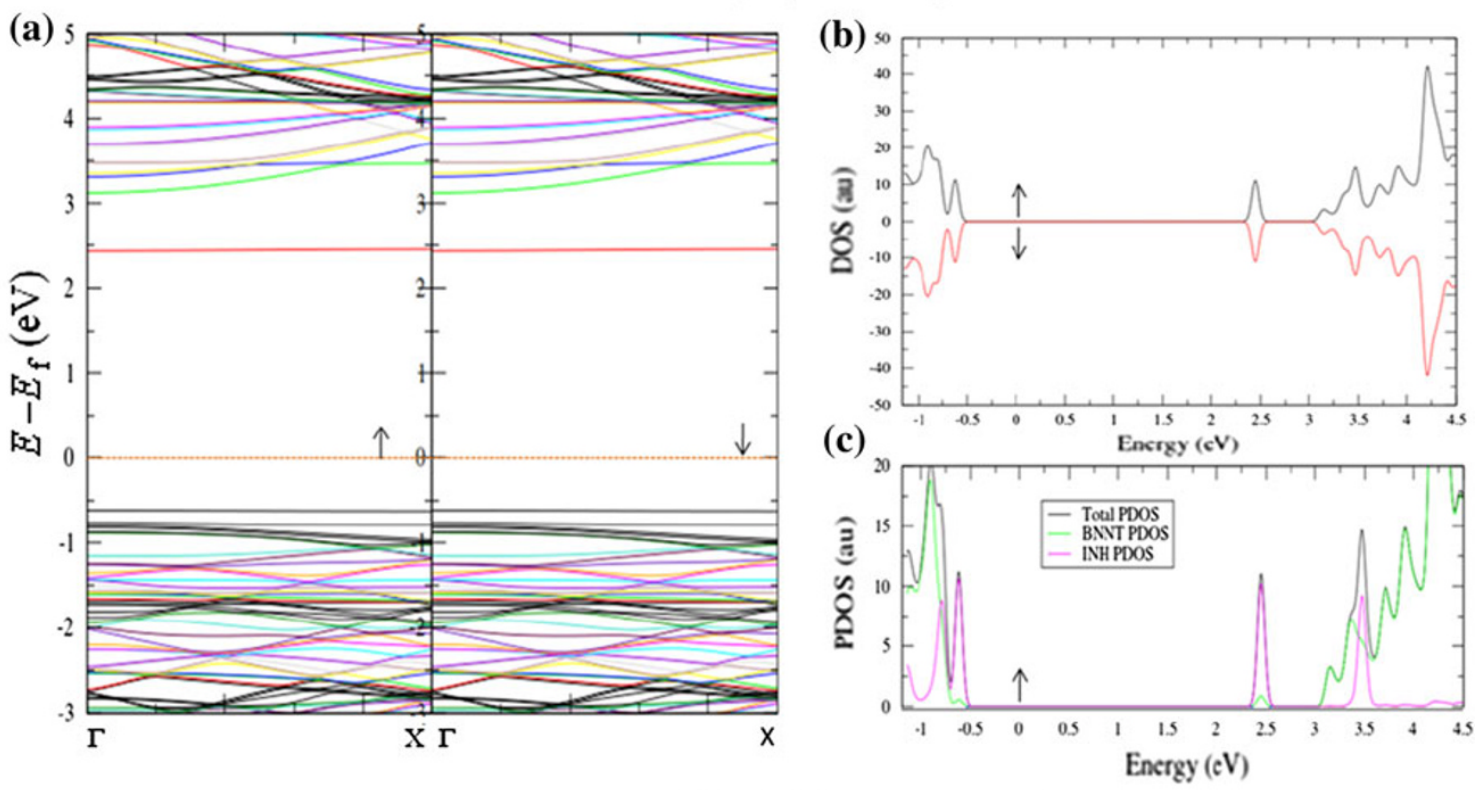

INH/(10,0) BNNTperpendicular site
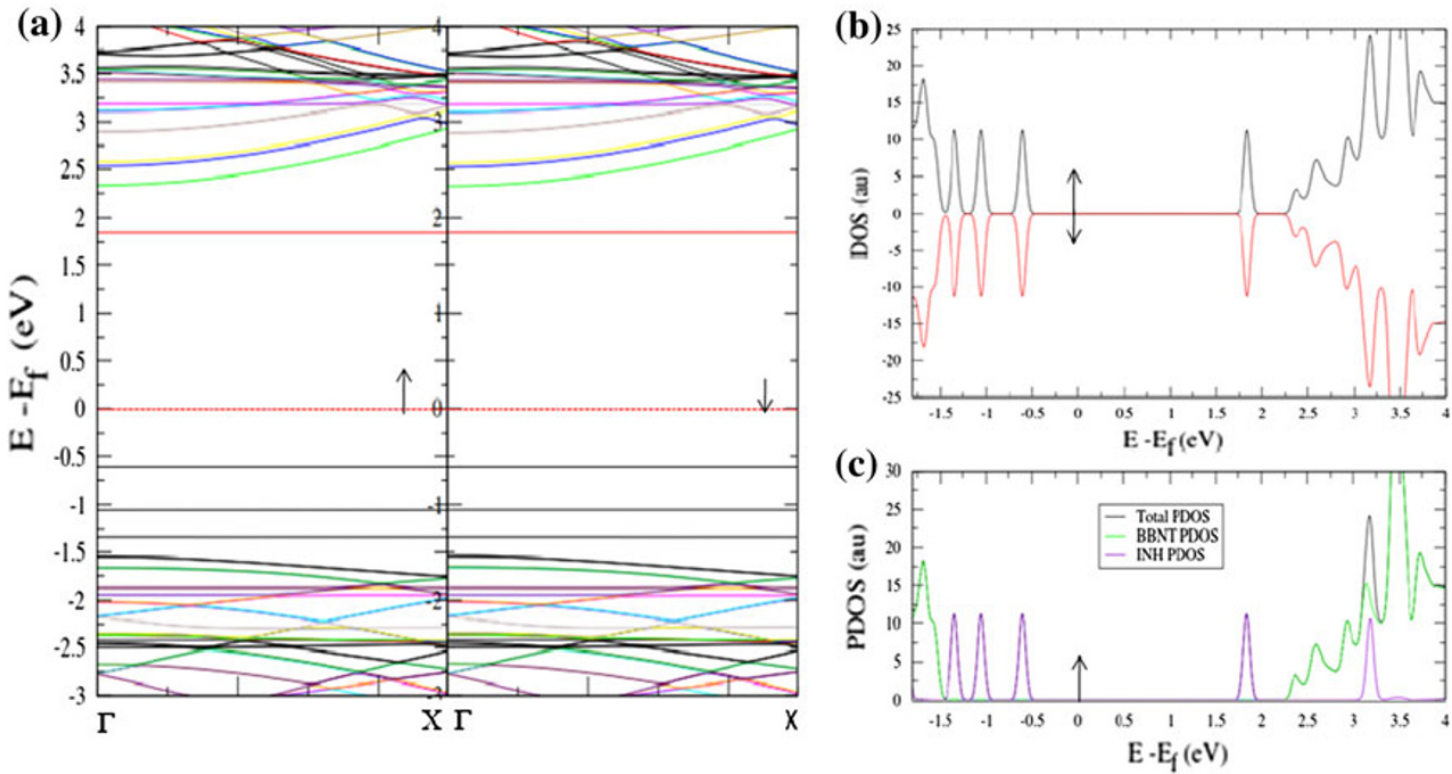

Fig. 7 Top the band structure (a), DOS (b) and PDOS (c) plots of INH adsorbed onto $(10,0)$ BNNT at the bridge site, and bottom the band structure (a), DOS (b) and PDOS (c) plots of INH adsorbed onto

adsorption onto both $(5,5)$ and $(10,0)$ BNNT in the vicinity of the Fermi level region which is validated from the band structure and PDOS plots.

Similarly, for INH adsorbed onto $(10,0)$ BNNT, the HOMO and LUMO plots for adsorption at the bridge site and perpendicular sites are shown in Fig. 9. The HOMO and LUMO plots for the other adsorption sites are given in the Supporting Information (SI, Fig. S8). For INH/(10,0) BNNT at the bridge site, the HOMO is highly delocalized around the INH molecule with partial localization of the
$(10,0)$ BNNT at the perpendicular site. The Fermi energy level is set to zero and indicated by the red dash line. The up and down arrows denote the up and down spins, respectively

electron density onto the nanotube sidewall at the close vicinity of the INH molecule. The LUMO, however, is more centred on the INH molecule with no major contribution from the (5,5) BNNT sidewall (Fig. 9b).

For the perpendicular adsorption of INH on to $(10,0)$ BNNT, the HOMO (Fig. 9c) and LUMO (Fig. 9d) are delocalized throughout the INH molecule with almost no contribution from the BNNT. Thus, for both $(5,5)$ and $(10,0)$ BNNTs, the adsorption of INH results in the delocalization of the electron density mostly onto the INH 
Fig. 8 Isosurface of the wavefunction of the HOMO and LUMO derived from the bands at the $\Gamma$ point, a HOMO of INH/ $(5,5)$ BNNT with INH at the N top site, $\mathbf{b}$ corresponding LUMO, c HOMO of INH/(5,5)

BNNT with INH at the perpendicular site, and $\mathbf{d}$ the corresponding LUMO
INH/(5,5) BNNT hollow site

(a)

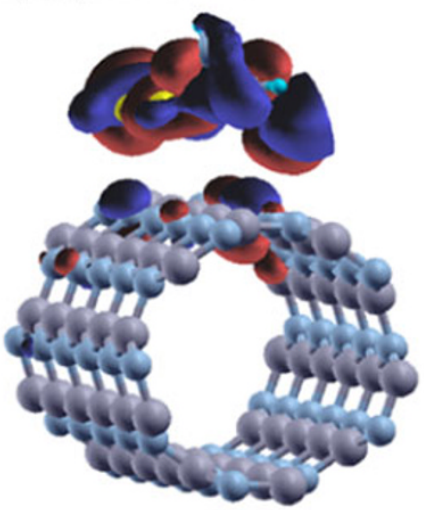

INH/(5,5) BNNT perpendicular

(c)
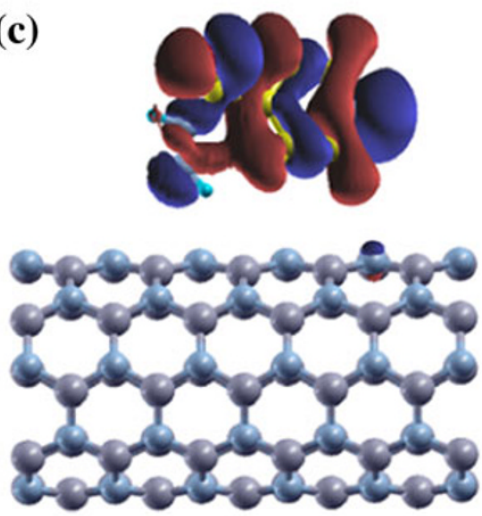

(b)

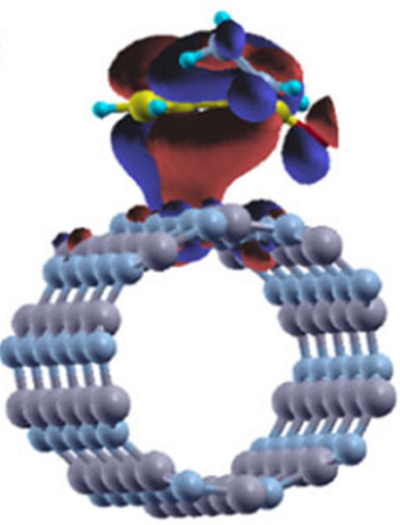

(d)
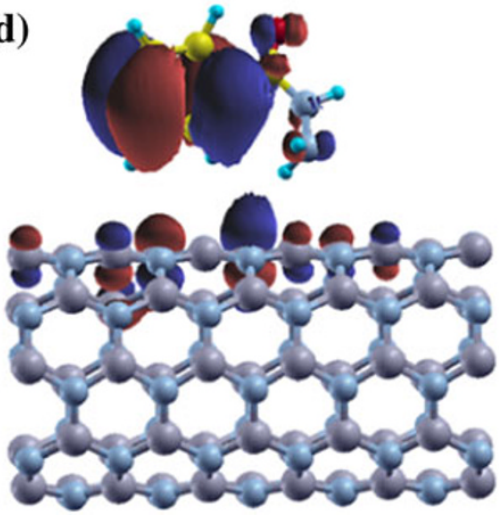

molecule with partial contributions coming from the nanotube. The same trend is observed for the other studied sites of adsorption of INH onto $(5,5)$ and $(10,0)$ BNNTs shown in the Supporting Information (SI, Figs. S7, S8). Compared to INH/(5,5) BNNT system, in case of INH/ $(10,0) \mathrm{BNNT}$, the charge localization over $(10,0) \mathrm{BNNT}$ is more prominent after adsorption of INH. The wavefunction and the PDOS plots collectively show that within the Fermi level region the electronic states and the contribution to the charge density are from the INH molecule itself, which justifies the study.

\section{Conclusion}

In conclusion, we have studied the structure and electronic properties of the noncovalent functionalization of $(5,5)$ and $(10,0)$ BNNTs with INH antitubercular drug molecule based on density functional calculations. Upon adsorption of INH onto $(5,5)$ BNNT, the hollow site is preferred for adsorption (adsorption energy of $-0.649 \mathrm{eV}$ ) whereas in $(10,0)$ BNNT, the bridge site is preferred at an adsorption energy value of $-0.738 \mathrm{eV}$. The inter-planar distances between INH and BNNT sidewall are about $3.175 \mathrm{eV}$ for $(5,5)$ BNNT and $3.218 \mathrm{eV}$ corresponding to $(10,0)$ BNNT.
The perpendicular adsorption is the least preferred as the interaction is not very significant with minimum $\pi-\pi$ stacking between the pyrazine ring of INH and BNNT sidewall. The trend in the adsorption with respect to the thermodynamic favourability is as follows:

INH hollow site $>$ INH N top $>$ INH B top $>$ INH bridge site $>$ INH perpendicular $(\mathrm{INH} /(5,5)$ BNNT system $)$

INH bridge site $>$ INH hollow site $>$ INH N top $>$ INH

B top $>$ INH perpendicular $(\mathrm{INH} /(10,0)$ BNNT system $)$

The functionalization of BNNT with INH brings about noticeable influence in the electronic property of BNNT and introduces new dispersionless bands (impurity or coupling states) within the HOMO-LUMO gap of pristine BNNT, resulting in the lowering of the energy gap and increase in reactivity of the system. The new impurity states are close to the Fermi level corresponding to the VBM (HOMO) of BNNT and also the CB region (LUMO) which is basically contributed from the INH molecule rather than the BNNT. The DOS and projected DOS (PDOS) plots clearly suggest that INH contributes towards the dispersionless bands in the Fermi level region and BNNT are low lying states having contribution away from 
Fig. 9 Isosurface of the wavefunction of the $\mathrm{HOMO}$ and LUMO derived from the bands at the $\Gamma$ point, a HOMO of INH/ $(10,0)$ BNNT with INH at the bridge site, $\mathbf{b}$ corresponding LUMO, c HOMO of INH/(10,0) BNNT with INH at the perpendicular site, and $\mathbf{d}$ the corresponding LUMO
$(10,0)$ BNNT INH bridge site

(a)

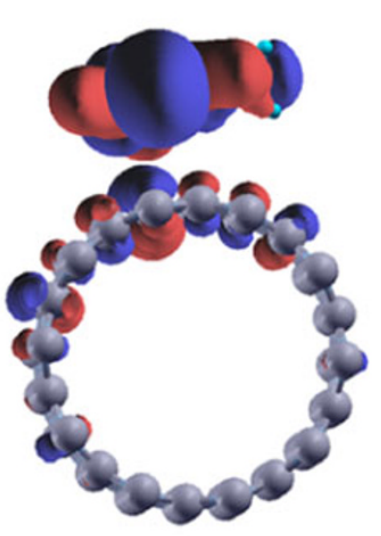

(b)

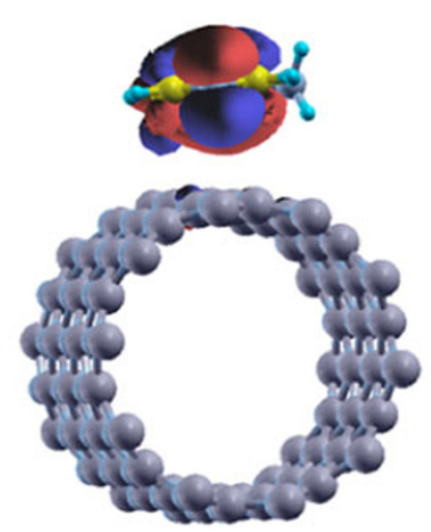

$(10,0)$ BNNTINH perpendicular site

(c)

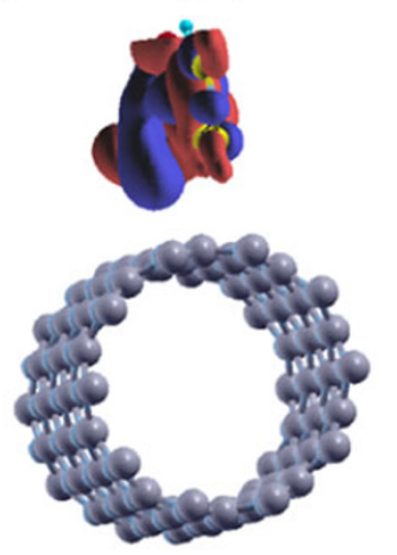

(d)
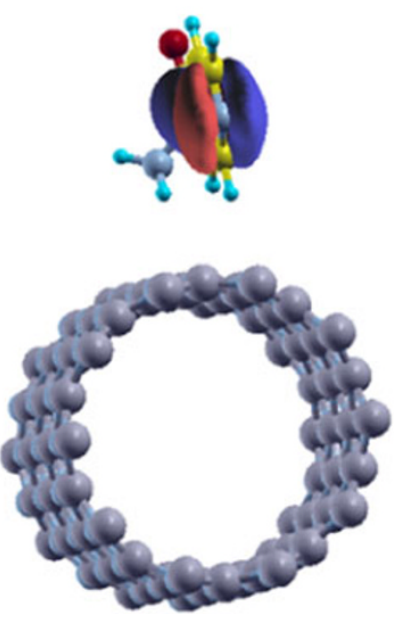

the Fermi region. Our studies thus contribute towards the basic understanding of the noncovalent functionalization of BNNT with antitubercular therapeutic molecules and how their subsequent adsorption with the semiconducting nanotubes can modify the electronic properties of the BNNT and can be foreseen as the good option for band gap engineering of the otherwise wide band gap nanotubes. This work supported with experimental studies can have significant impetus in tuberculosis chemotherapy.

Acknowledgments All the calculations have been performed using the computational facilities at Theoretical Chemistry Unit (TSU), JNCASR, Bangalore, India under the guidance of Prof. Swapan K. Pati. The assistance and help endowed by Prof CNR Rao for carrying out the research work at JNCASR are kindly acknowledged. NS would like to thank the fellow research workers of Quantum: Molecules to Materials Unit for their unprecedented help and guidance in carrying out the work. NS and RCD would like to acknowledge The Department of Science and Technology, New Delhi, India for funding the project and providing the necessary grant in aid.

Open Access This article is distributed under the terms of the Creative Commons Attribution License which permits any use, distribution, and reproduction in any medium, provided the original author(s) and the source are credited.

\section{References}

Akdim B, Kim SN, Naik RR, Maruyama B, Pender MJ, Pachter R (2009) Understanding effects of molecular adsorption at the single-wall boron nitride nanotube interface from density functional theory calculations. Nanotechnology 20(355705):1-8

Blase X, Rubio A, Louie SG, Cohen ML (1994) Stability and band gap consistency of boron nitride nanotubes. Europhys Lett 28:335-340

Chen X, Wu P, Rousseas M, Okawa D, Gartner Z, Zettl A, Bertozzi CR (2009) Boron nitride nanotubes are noncytotoxic and can be functionalized for interaction with protein and cells. J Am Chem Soc 131:890-891

Chopra NG, Luyken RL, Cherrey K, Crespi VH, Cohen ML, Louie SG, Zettl A (1995) Boron nitride nanotubes. Science 269: 966-967

Dessen A, Quemard A, Blanchard JS Jr, Jacobs WR, Sacchettini JC (1995) Crystal structure and function of the isoniazid target of Mycobacterium tuberculosis. Science 267:1638-1641

Favila A, Gallo M, Glossman-Mitnik D (2007) CHIH-DFT determination of the molecular structure, infrared spectra, UV spectra and chemical reactivity of three antitubercular compounds: rifampicin, isoniazid and pyrazinamide. J Mol Model 13: 505-518

Gao Z, Zhi C, Bando Y, Golberg D, Serizawa T (2011) Noncovalent functionalization of disentangled boron nitride nanotubes with flavin mononucleotides for strong and stable visible-light 
emission in aqueous solution. ACS Appl Mater Interfaces 3:627-632

Golberg D, Han W, Bando Y, Bourgeois L, Kurashima K, Sato T (2001) Synthesis and characterization of ropes made of $\mathrm{BN}$ multi-walled nanotubes. Scipta Mater 44:1561-1565

Gou G, Pan B, Shi L (2010) Noncovalent functionalization of BN nanotubes with perylene derivative molecules: an ab initio study. ACS Nano 4:1313-1320

Helland A, Wick P, Koehler A, Schmid K, Som C (2007) Reviewing the environmental and human health knowledge base of carbon nanotubes. Environ Health Perspect 115:1125-1131

Kleinman L, Bylander DM (1982) Efficacious form for model pseudopotentials. Phys Rev Lett 48:1425-1428

Monkhorst HJ, Pack JD (1976) Special points for Brillouin-zone interactions. Phys Rev B 13:5188-5192

Mukhopadhyay S, Gowtham S, Scheicher RH, Pandey R, Karna SP (2010) Theoretical study of physisorption of nucleobases on boron nitride nanotubes: a new class of hybrid nano-biomaterials. Nanotechnology 21(165703):1-6

Pewdew JP, Burke K, Ernzerhof M (1996) Generalized gradient approximation made simple. Phys Rev Lett 77:3865-3868
Rubio A, Corkill JL, Cohen ML (1994) Theory of graphitic boron nitride nanotubes. Phys Rev B 49:5081-5084

Scroeder EK, de Souza ON, Santos DS, Blanchard JS, Basso LA (2002) Drugs that inhibit mycolic acid biosynthesis in Mycobacterium tuberculosis. Curr Pharm Biotechnol 3:197-225

Troullier N, Martins JL (1991) Efficient pseudopotentials for planewave calculations. Phys Rev B 43:1993-2006

Wang WL, Bando Y, Zhi CY, Fu WY, Wang EG, Golberg D (2008) Aqueous noncovalent functionalization and controlled nearsurface carbon doping of multiwalled boron nitride nanotubes. J Am Chem Soc 130:8144-8145

Zhao J-X, Ding Y (2010) Theoretical study of noncovalent functionalization of BN nanotubes by various aromatic molecules. Diam Relat Mater 19:1073-1077

Zhi C, Bando Y, Tang C, Golberg D (2006) Engineering the electronic structure of boron nitride nanotubes bycovalent functionalization 74:153413-153414

Zhi CY, Bando Y, Tang CC, Huang Q, Golberg D (2008) Boron nitride nanotubes: functionalization and composites. J Mater Chem 18:3900-3908 